\title{
EMISIONES BIOGÉNICAS DE MONOTERPENOS EN EL PARQUE NACIONAL NATURAL TAYRONA, SANTA MARTA (COLOMBIA)
}

\author{
Tomás Rafael Bolaño Ortiz ${ }^{1}$ \\ Yiniva Camargo Caicedo ${ }^{2}$ \\ Andrés Mauricio Vélez-Pereira ${ }^{3}$ \\ Recibido el 19 de marzo de 2012, aprobado el 13 de septiembre de 2014, \\ actualizado el 1 noviembre de 2014
}

DOI: 10.17151/luaz.2015.40.8

\section{RESUMEN}

Los monoterpenos y otros compuestos orgánicos volátiles emitidos por la vegetación (emisiones biogénicas) juegan un papel significativo en la química troposférica por su participación en la formación de oxidantes fotoquímicos (p.e. ozono troposférico). Es así como la estimación de emisiones biogénicas resulta útil para establecer valores aproximados de la concentración, fundamental en la elaboración de programas de mejoramiento de calidad del aire. El objetivo del trabajo consistió en estimar las emisiones biogénicas de monoterpenos en el Parque Nacional Natural Tayrona, seleccionado por contar con áreas de vegetación autóctona de la región tropical con escasa o nula intervención antropogénica. Se aplicaron sensores remotos para la clasificación de cobertura vegetal, obteniendo la distribución espacial de la vegetación típica e identificando las familias de mayor dominancia. Las emisiones de monoterpenos se calcularon en $3,54 \times 10^{4} \mathrm{~kg} \mathrm{~d}^{-1}$, representadas $60 \%$ por la familia Leguminosae, $37,4 \%$ por la familia Euphorbiaceae, 2,4\% por la familia Anacardiaceae y menos de $1 \%$ por las familias Rubiaceae y Polygonaceae. Durante las horas de sol en el área del parque (de las 6 a las 18 horas) se registraron las mayores temperaturas diarias y las más altas tasas de emisión de monoterpenos (59,6\%); la cuarta parte de las emisiones ocurren entre las 10 y las 15 horas $(24,2 \%)$, periodo en el que la radiación solar presenta máximos diarios y establece condiciones críticas para la formación de oxidantes fotoquímicos. La influencia de los vientos en la distribución espacio-temporal de las emisiones en el ambiente y la información de la rosa de vientos, evidencia que el $26 \%$ de las masas de aire proceden del $\mathrm{N}$ y el $15 \%$ del NE en el último trimestre del año, situación que podría favorecer la dispersión de monoterpenos a centros urbanos próximos al parque natural, pudiendo alterar la química atmosférica local con la formación de oxidantes fotoquímicos.

\section{PALABRAS CLAVE}

Emisiones biogénicas, monoterpenos, oxidantes fotoquímicos, vegetación.

\section{MONOTERPENES BIOGENIC EMISSIONS IN THE TAYRONA NATURAL PARK, SANTA MARTA (COLOMBIA)}

\begin{abstract}
Monoterpenes and other volatile organic compounds emitted by vegetation (biogenic emissions) play a significant role in tropospheric chemistry because of their participation in the formation of photochemical oxidants (i.e. troposfheric ozone). This is how the estimation of biogenic emissions is useful for determining approximate values of monoterpenes concentration, which is essential in developing programs to improve air quality. The aim of this work was to estimate biogenic emissions of
\end{abstract}


monoterpenes in the Tayrona National Park, that was selected because of the presence of native vegetation of the tropics with little or no human interference. Remote sensing was applied for classification of vegetation, coverage obtaining the spatial distribution of the typical vegetation and identifying the most dominant families. Monoterpenes emissions were estimated at $3.54 \times 104 \mathrm{~kg} \mathrm{~d}-1$, represented $60 \%$ of the Leguminosae family, $37.4 \%$ of the Euphorbiaceae family, $2.4 \%$ of the Anacardiaceae family and less than $1 \%$ of Rubiaceae and Polygonaceae families. During daylight hours in the park area (from 6:00 a.m. to 6:00 p.m.) the highest daily temperatures were registered and the highest emission rates of monoterpenes $(59.6 \%)$; one fourth of the emissions occur between 10:00 a.m. and 3:00 p.m. $(24.2 \%)$, period in which solar radiation presents daily maximums and establishes critical conditions for the formation of photochemical oxidants. The influence of winds on the spatial-temporal distribution of emissions shows that $26 \%$ of the air mass come from the $\mathrm{N}$ and $15 \%$ of $\mathrm{NE}$ in the last quarter of year, a situation that could favor the spread of monoterpenes to urban centers near the natural park, which could alter the local atmospheric chemistry with the formation of photochemical oxidants.

\section{KEY WORDS}

Biogenic emissions, monoterpenes, photochemical oxidants, vegetation.

\section{INTRODUCCIÓN}

Las zonas industriales y áreas urbanas se han constituido en los responsables principales de la contaminación atmosférica debido a la emisión de material particulado y Compuestos Orgánicos Volátiles (COV) producto de las actividades antropogénicas. En los últimos años, se ha centrado el interés en el estudio de los COV por su capacidad de actuar como sustancias precursoras en el proceso de formación de contaminantes fotoquímicos, dentro de los que se destaca el ozono troposférico (Camargo et al., 2010).

La emisión de COV a la atmósfera, proceden tanto de origen natural como antropogénico. Las emisiones biogénicas se refieren en su mayoría a hidrocarburos del grupo de los COVs emitidos por fuentes naturales, dentro de las que se destacan los provenientes de la vegetación (Camargo et al., 2010). Las cuales al ser liberadas a la atmósfera reaccionan con otros compuestos formando parte activa en la química atmosférica, registrando contribuciones de hasta dos terceras partes de las emisiones globales, excediendo incluso a las emisiones antropogénicas (Guenther et al., 1994; Sabillón, 2001).

Los inventarios de emisiones biogénicas al igual que los inventarios de emisiones antropogénicas, suelen utilizarse como datos de entrada para modelos de calidad del aire, que tienen como finalidad predecir las concentraciones de contaminantes primarios y secundarios, al simular diversas condiciones meteorológicas y el comportamiento de las fuentes. Los resultados obtenidos son utilizados para evaluar diferentes medidas de control y aplicar estrategias para disminuir los niveles de determinados contaminantes (Velasco \& Bernabé, 2004).

Los compuestos que hacen parte de las emisiones biogénicas participan en la generación de ozono troposférico, resultando objeto de investigación en diferentes lugares del mundo, como lo demuestran los estudios realizados en la zona continental de Estados Unidos (Tao et al., 2003), el estudio realizado por Derognat 
et al. (2003) en París (Francia) y los resultados presentados por Thunis \& Cuvilier (2000) para la zona mediterránea, los inventarios presentados por Velasco (2003) para el Valle de México, Wang, Yuhua \& Shuyu (2003) para Beijing (China) y el inventario desarrollado por Xu, Wesely \& Pierce (2002), mediante el uso de observaciones satelitales, para la zona oriental de Estados Unidos.

Los monoterpenos $\left(\mathrm{C}_{10} \mathrm{H}_{\mathrm{x}}\right)$ junto al isopreno son los compuestos orgánicos volátiles biogénicos (COVB) que dominan las emisiones a escala global (Guenther et al., 1995; Räisänen, Ryyppö \& Kellomäki, 2009), y son miembros importantes de la familia de los isoprenoides (o terpenoides) (McGarvey \& Croteau, 1995). Estos compuestos normalmente se pueden almacenar en órganos especializados de las hojas y tallos, lo que contribuye a que parte del carbono que se ha producido en los tejidos vegetales mediante diversos procesos fisiológicos retorne hacia la atmósfera. Esta emisión es producida principalmente por la difusión, debido a un gradiente de presión de vapor desde los compartimentos celulares con concentraciones relativamente altas hacia el aire circundante a las hojas.

Los monoterpenos pueden reaccionar con el vapor de agua para dar lugar a ácidos orgánicos débiles que son depositados en las superficies terrestres con el agua de Iluvia afectando la química del suelo (Sabillón, 2001; Velasco \& Bernabé, 2004). Por otro lado, las reacciones fotoquímicas en la atmósfera en presencia de COV $(\mathrm{RH})$ y de radicales hidroxilo $(\mathrm{OH})$, producen una serie de reacciones que, mediante radicales peroxialquilo $\left(\mathrm{RO}_{2}\right)$, convierten el $\mathrm{NO}$ en $\mathrm{NO}_{2}$ sin consumir una molécula de ozono, dando como resultado una acumulación de ozono en la troposfera (ver Figura 1), Este incremento de la concentración de ozono troposférico debido a las emisiones de COVB, ocurre cuando el aire está contaminado, ya que cuando la concentración de NOx es baja, como es el caso de las regiones remotas, dichas emisiones pueden provocar un descenso en la concentración de ozono (Fehsenfeld et al., 1992).

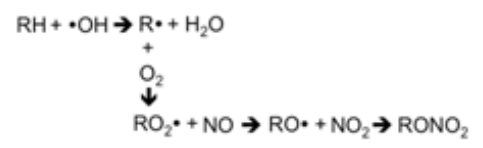

Figura 1. Esquema general de las reacciones fotoquímicas de los COV en la atmósfera.

(Adaptado de Sabillón, 2001).

En general, los terpenos emitidos reaccionan rápidamente con el radical $\mathrm{OH}$, disminuyendo la concentración de este, aunque favorecen el aumento de las concentraciones de los radicales $\mathrm{HO}_{2}$ y $\mathrm{RO}_{2}$. La reacción de oxidación del a-pineno y el $\Delta^{3}$-careno por el radical $\mathrm{OH}$ es la más conocida, dando como resultado la formación de un ceto-aldehído $\left(\mathrm{P}_{1} \mathrm{CHO}\right)$, con el a-pineno se genera el pinonaldehído (Arey et al., 1990; Janson, 1992).

En conclusión, las principales consecuencias que resultan de los complejos mecanismos de reacción que comprenden la oxidación de los monoterpenos y el isopreno se resumen así (Cremades \& Ribes, 1997):

- La presencia de compuestos biogénicos en una atmósfera que contiene óxidos de nitrógeno puede incrementar la formación de ozono.

- Este tipo de emisiones controlan la concentración de radicales hidroxilo en la troposfera.

- Se estima que la foto-oxidación de terpenos puede ser la mayor fuente del CO troposférico, un gas importante que influye en la concentración del radical $\mathrm{OH}$ en la atmósfera. 
- Las emisiones biogénicas tienen una alta importancia en la formación de nitratos orgánicos, los cuales pueden atrapar los NOx, permitiendo su transporte a través de largas distancias.

- La oxidación de algunos de estos compuestos puede dar lugar a aerosoles orgánicos y contribuir así a un aumento en la formación de nieblas.

- Los monoterpenos pueden reaccionar con el vapor de agua, generando ácidos orgánicos débiles que son depositados en la superficie terrestre con el agua de lluvia, afectando la química del suelo.

\section{METODOLOGÍA}

\section{Área de estudio}

La zona de estudio corresponde al Parque Nacional Natural Tayrona (PNNT), en el norte de Colombia, se encuentra ubicada en las coordenadas $11^{\circ} 21^{\prime}-11^{\circ} 16 \mathrm{~N}$ y $73^{\circ} 53^{\prime}-74^{\circ} 13^{\prime} \mathrm{W}$ (Figura 2), y constituye una reserva natural con amplias áreas forestales de vegetación tropical protegida contra actividades antropogénicas, por lo que estimar las emisiones de monoterpenos en el PNNT resulta interesante en la medida en que se inicia el proceso donde se puede establecer el aporte de la vegetación a las concentraciones de fondo en esta área. Por lo anterior, el objetivo general de este trabajo consistió en estimar las emisiones biogénicas de monoterpenos en el PNNT en Santa Marta (Colombia).

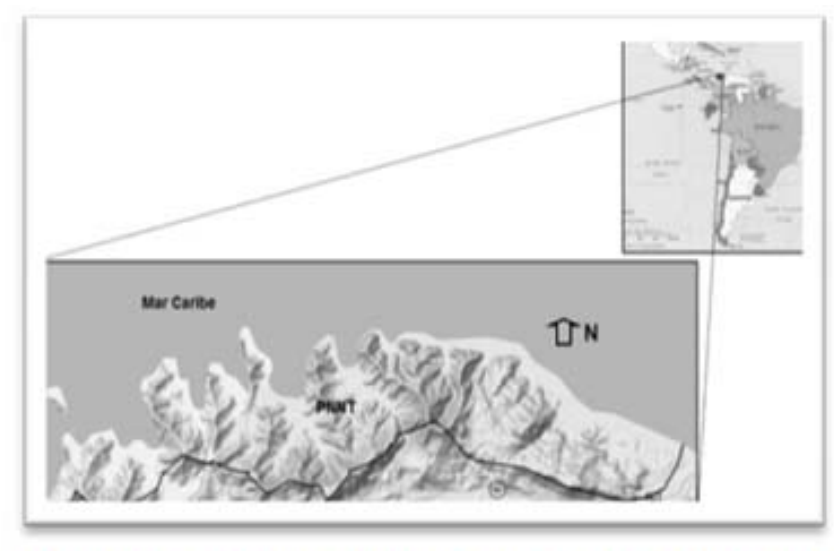

Figura 2. Ubicación geográfica del área de estudio en Colombia y Suramérica.

\section{Identificación de la cobertura vegetal y uso del suelo}

La identificación se realizó a partir de los segmentos combinados de una imagen satelital LandSat TM tomada del área de estudio el 18 de septiembre de 2007 (Figura 3), el análisis de mapas de uso del suelo del Instituto Geográfico Agustín Codazzi (IGAC) y los estudios de distribución vegetal (Puentes, 2002). 


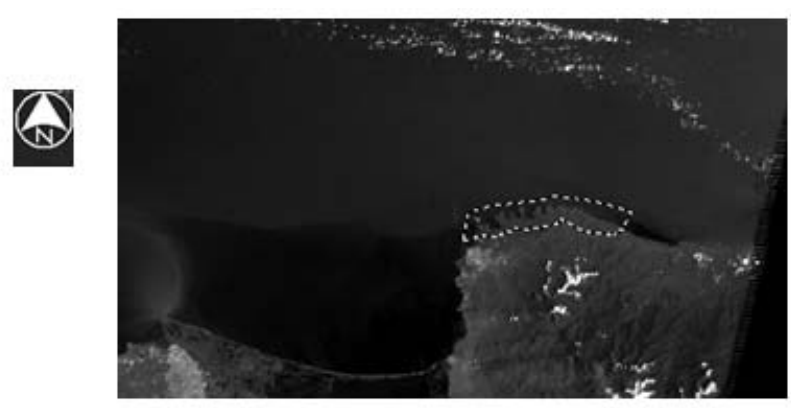

Figura 3. Fragmento de imagen de satélite LandSat TM capturada el 18/09/07 (USGS

Global Visualization Viewer-GLOVIS-).

Se identificaron las principales características topográficas y geográficas, características del suelo y distribuciones de las principales especies vegetales. Se realizó una clasificación no supervisada, que incluyó el análisis de zonas de entrenamiento con ayuda del conocimiento previo, luego se usó el método de agrupación de pixeles de una imagen según su similitud espectral. En el procesamiento de la imagen fue utilizado el software Erdas Imagine, aplicando el algoritmo isodata, y la fórmula de la distancia mínima espectral para formar cúmulos (cluster), donde las firmas espectrales fueron automáticamente generadas por este algoritmo.

\section{Verificación y registro de información de campo}

Se realizó la verificación en campo, el día 19 de octubre de 2009, para establecer la precisión de la clasificación efectuada con las imágenes satelitales y mejorar la identificación de comunidades típicas de la vegetación (Chuvieco, 2008), áreas de cobertura vegetal, distribución de especies, densidad de biomasa, promedio de diámetro a la altura del pecho (DAP) y promedio de altura. Se visitaron cuatro emplazamientos, que se indican en la Tabla 1.

Tabla 1. Relación georreferenciada de los sitios visitados

\begin{tabular}{cll}
\hline Punto & Sitios visitados & Coordenadas \\
\hline \multirow{2}{*}{1} & Kalashe & $11^{\circ} 17^{\prime} 07,6^{\prime \prime} \mathrm{N}$ \\
& & $74^{\circ} 05^{\prime} 09,3^{\prime \prime} \mathrm{W}$ \\
2 & \multirow{2}{*}{ Neguanje oeste } & $11^{\circ} 18^{\prime} 47,9^{\prime \prime} \mathrm{N}$ \\
& & $74^{\circ} 05^{\prime} 11,9^{\prime \prime} \mathrm{W}$ \\
3 & & $11^{\circ} 19^{\prime} 39,3^{\prime \prime} \mathrm{N}$ \\
& & $74^{\circ} 05^{\prime} 57,5^{\prime \prime} \mathrm{W}$ \\
4 & Neguanje este & $11^{\circ} 19^{\prime} 45,3^{\prime \prime} \mathrm{N}$ \\
& & $74^{\circ} 04^{\prime} 39,3^{\prime \prime} \mathrm{W}$ \\
\hline
\end{tabular}

Se captaron muestras de material foliar de las plantas con la finalidad de hacer la posterior clasificación taxonómica en el Herbario de la Universidad del Magdalena, organismo que cuenta con un registro nacional de colecciones biológicas, 
identificado con el No. 032 del Instituto de Investigación de Recursos Biológicos Alexander von Humboldt, miembro de la Asociación Colombiana de Herbarios y registro UTMC en el Index Herbariorum.

\section{Clasificación de la vegetación típica}

Las características de la vegetación encontrada con las imágenes satelitales, además de la información complementaria (Puentes, 2002) y verificada con las salidas de campo, permitió efectuar la identificación de aquellas plantas que según la literatura consultada emiten monoterpenos (Lancaster University, 2009), estableciendo su ubicación y clasificación taxonómica, a partir de las claves de la sistemática botánica para darle a cada especie y familia su nombre genérico.

\section{Emisiones de monoterpenos por familia}

La vida media de los monoterpenos corresponde generalmente a pequeños periodos de tiempo, su respuesta para cortos periodos a la luz y la temperatura (short term), han sido ampliamente estudiadas para muchas especies vegetales y compuestos, siendo algunas descritas en modelos matemáticos (Tingey et al., 1980; Guenther, Monson \& Fall, 1991; Schuh et al., 1997; Staudt \& Bertin, 1998; Sabillón, 2001). Todos los estudios mencionados evidencian y correlacionan la influencia de factores ambientales en periodos cortos de tiempo.

Para el caso de los monoterpenos, se estableció un modelo de emisiones en función de la temperatura (Tingey et al., 1980), manifestándose que existen plantas como las de la familia Pinaceae que tienen estructuras en las que almacenan este terpenoide y generan una fuente permanente de emisión por la hoja (Schindler \& Kotzias, 1989; Lerdau, Litvak \& Monson, 1994).

Por consiguiente, se prevé que la emisión de monoterpenos es ocasionada especialmente por la volatilización en los órganos de depósito, es decir, solo se asumen los resultados de la temperatura sobre la presión de vapor del gas que se encuentra en el tejido de la planta y la resistencia de transporte de difusión (Tingey et al., 1991; Simon et al., 1994; Monson et al., 1995).

Teniendo en cuenta que la emisión de monoterpenos solo varía con la temperatura, se ha establecido el siguiente modelo, recogido en las expresiones (1) y (2) aplicable para aquellas plantas con capacidad de almacenar monoterpenos (Tingey et al., 1980; Guenther, Zimmerman \& Harley, 1993):

$$
E_{i j}=A r \times \rho_{j} \times A_{j} \times F_{j} M_{t}
$$

\section{Donde:}

Eij: flujo de emisión calculado en $\mu \mathrm{g} \mathrm{h}^{-1}$, para los monoterpenos (i) emitidos por la especie o familia j.

Ar: cociente entre el área árboles (especie o familia j) y el área región ocupada por esta $\left(\mathrm{m}^{2} \mathrm{~m}^{-2}\right)$.

$\rho \mathrm{J}$ : densidad de biomasa $\left(\mathrm{g} \mathrm{m}^{-2}\right)$.

Aj: área región $\left(\mathrm{m}^{2}\right)$.

Fj: factor de emisión estándar de monoterpenos para especie o familia j $\left(\mu \mathrm{g} \mathrm{g}^{-1} \mathrm{~h}^{-1}\right)$.

Mt: expresión usada para realizar la corrección por temperatura (Tingey et al., 1980). 
Calculando Mt según la ecuación 2

$$
M_{t}=e^{[\beta(T-T)]}
$$

Donde:

T: Temperatura de la hoja en $\mathrm{K}$.

Ts: Temperatura estándar (303 K).

$\beta$ : Coeficiente que establece la dependencia de la emisión de la temperatura, varía de 0,057 a $0,144 \mathrm{~K}^{-1}$, siendo utilizado un valor típico de $0,09 \mathrm{~K}^{-1}$ debido a que empíricamente es el perteneciente al $50 \%$ de las especies.

\section{Estimación de la cantidad de emisiones biogénicas a partir de monoterpenos}

El inventario fue realizado teniendo como día base el 18 de septiembre de 2007, fecha en la que fue captada la imagen de satélite por el sensor Thematic Mapper (TM) de LandSat, usada en la clasificación espacial del área de estudio.

Así mismo los datos de temperatura corresponden a históricos del Instituto de Hidrología, Meteorología y Estudios Ambientales de Colombia (IDEAM) para ese año. En esta etapa se procedió a compilar la información verificada en campo, datos tomados de la literatura y el resultado del procesamiento de la imagen de satélite del área de estudio usando las ecuaciones 1 y 2 para los datos de cada familia. No se tomaron datos más recientes por motivos de disponibilidad por parte de las instituciones encargadas de realizar el registro de datos climatológicos y meteorológicos. En la Figura 4 se resume la metodología empleada para la estimación de las emisiones de monoterpenos. 


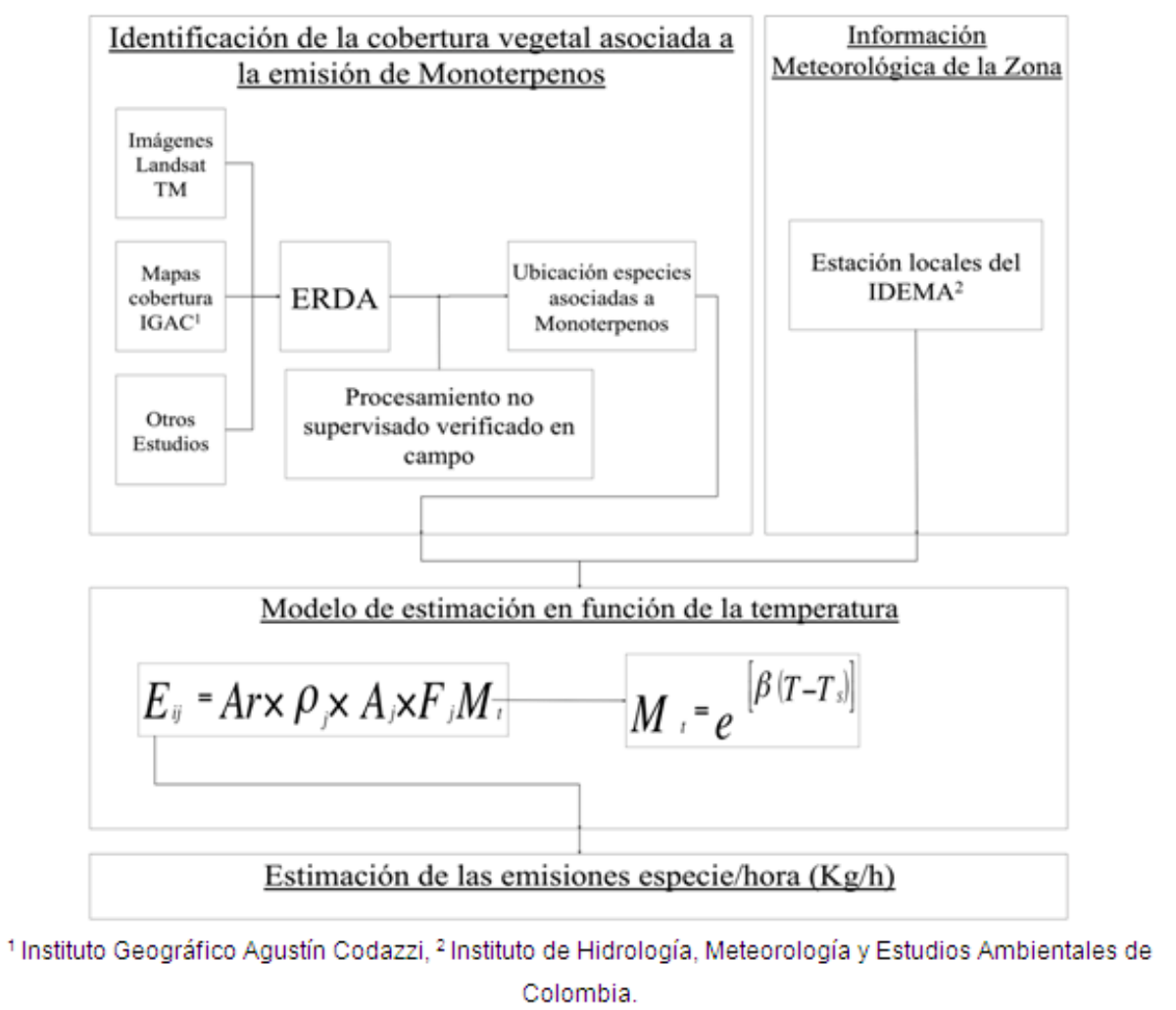

Figura 4. Resumen de la metodología para la estimación de las emisiones de monoterpenos en el PNNT.

\section{Criterios de calidad}

La aplicación de unos criterios de calidad es una herramienta indispensable en cualquier inventario de emisiones. Sin importar el tipo de inventario de que se trate es necesario usarlo. Su cantidad y el enfoque pueden ser versátiles y dependen del propósito específico del inventario. En este caso se tuvieron en cuenta las siguientes consideraciones con la finalidad de minimizar los sesgos:

- Se usó una lista verificable de fuentes bibliográficas que confirma que han sido incluidas en el inventario las diversas categorías. Estas fueron seleccionadas en función de las condiciones locales, es decir, solo se utilizaron aquellas relevantes para el tipo de vegetación presente en el área de estudio.

- Han sido revisados los resultados y verificados los datos de cada actividad buscando aquellos valores que estén fuera de lugar, antes de calcular las emisiones.

- Se verificó el cálculo de emisión (ecuaciones 1 y 2) para garantizar que se ha hecho de manera apropiada. Para esto se utilizó una hoja de cálculo electrónica.

- Han sido comparados los resultados del inventario con los de otros inventarios en regiones tropicales y subtropicales. 


\section{RESULTADOS Y DISCUSIONES}

Se realizó una clasificación supervisada de la cobertura vegetal típica con emisiones de monoterpenos, y con las áreas de entrenamiento visitadas se pudo estimar la distribución en el PNNT de las familias Anacardiaceae, Leguminosae, Rubiaceae, Euphorbiaceae y Polygonaceae, las cuales engloban 23 especies con potencial de generar emisiones biogénicas del compuesto estudiado, de acuerdo con los factores de emisión (Lancaster University, 2009).

Se estableció la presencia de estas familias vegetales en el territorio terrestre del PNNT, la familia Euphorbiaceae en 4810,5 ha (37\%) es la más representativa, seguida por la familia Anacardiaceae, la cual está presente en 3398,58 ha $(26,1 \%)$. La familia Polygonaceae en 2785.95 ha $(21,4 \%)$, la familia Leguminosae en 1614,61 ha $(12,4 \%)$ y la familia Rubiaceae en 388,35 ha $(3 \%)$.

La Tabla 2 expone los atributos más representativos de las familias vegetales utilizados para realizar los cálculos de emisiones horarias de monoterpenos en el PNNT. Sobresaliendo así, la familia Euforbiaceae por el área ocupada, la familia Leguminosae por su abundancia y factor de emisión de monoterpenos, y la familia Rubiaceae por la densidad de biomasa foliar.

Tabla 2. Principales características de las familias vegetales estudiadas en el PNNT

\begin{tabular}{cccc}
\hline Familia & $\begin{array}{c}\text { Abundancia } \\
\text { (árboles/ha) }\end{array}$ & $\begin{array}{c}\text { Densidad } \\
\text { de biomasa } \\
\text { foliar }\left(\mathrm{g} \mathrm{m}^{-2}\right)\end{array}$ & $\begin{array}{c}\text { Factores } \\
\text { de emisión } \\
\left(\mathrm{g} \mathrm{g} \mathrm{g}^{-1} \mathrm{~h}^{-1}\right)\end{array}$ \\
\hline Anacardiaceae & 175 & 230 & 0,6 \\
Leguminosae & 560 & 215 & 4,4 \\
Euphorbiaceae & 460 & 185 & 2,6 \\
Rubiaceae & 300 & 247 & 0,1 \\
Polygonaceae & 60 & 159 & 0,1 \\
\hline
\end{tabular}

\section{Análisis de tendencias de emisiones de monoterpenos}

En el análisis de las emisiones de monoterpenos en el área de estudio se utilizaron los datos de temperatura horaria del día 18 de septiembre de 2007, tomados por una estación climatológica ordinaria del IDEAM ubicada en el PNNT. Estos valores fueron seleccionados por encontrarse dentro de los valores medios típicos (IDEAM, 2007a, 2007b, 2007c) registrados en los últimos 24 años. Además, estudios realizados por este instituto sobre la distribución espacial de la temperatura en el departamento del Magdalena, evidencian que en la zona registra valores superiores a $24^{\circ} \mathrm{C}$, por tanto estos valores son considerados en este estudio como datos de temperatura representativos para el PNNT. Teniendo en cuenta lo anterior, todos los registros fueron analizados a fin de descartar aquellos claramente erróneos. Se asumieron como válidos aquellos registros que variaban dentro del intervalo 22 a $45^{\circ} \mathrm{C}$.

La Tabla 3 muestra la evolución diaria de los valores de emisión para las especies estudiadas. La evolución horaria de las emisiones durante el día 18 de septiembre de 2007, para el caso de monoterpenos se presentan durante las 24 horas, siendo sus tasas de emisión menores en las primeras horas del día (cuando la temperatura ambiente es baja) y mayores durante las horas del mediodía. En general, estas 
emisiones varían entre $1144,74 \mathrm{~kg} \mathrm{~h}^{-1}$ y $1027,55 \mathrm{~kg} \mathrm{~h}^{-1}$ durante las primeras horas del día, y sube hasta $2394,50 \mathrm{~kg} \mathrm{~h}^{-1}$ hacia el mediodía.

El 59,6\% del total diario de las emisiones biogénicas ocurren entre las 06 a 19 horas. Cerca de una cuarta parte $(24,2 \%)$ ocurren entre las 10 y las 14 horas, y en la madrugada (04-05 horas) se alcanzan sus menores niveles de emisión $(2,8 \%)$. El alto peso porcentual del total diario de las emisiones de monoterpeno $(40,1 \%)$ se genera entre las 9 y 16 horas, periodo en que tanto la temperatura como la radiación solar son más altas, y corresponden a condiciones críticas para la formación del $\mathrm{O}_{3}$ troposférico.

Tabla 3. Emisiones biogénicas de monoterpenos para las condiciones del PNNT el

18 de septiembre de 2007

\begin{tabular}{|c|c|c|c|c|c|c|c|}
\hline \multirow{3}{*}{ Hora } & Familia & Familia & Familia & Familia & Familia & \multirow{2}{*}{\multicolumn{2}{|c|}{ Total }} \\
\hline & Anacardiaceae & Leguminosae & Rubiaceae & Euphorbiaceae & Polygonaceae & & \\
\hline & \multicolumn{5}{|c|}{$\mathrm{kg} \mathrm{h}^{-1}$} & & $\%$ \\
\hline $00-01$ & 27,74 & 686,50 & 0,22 & 428,15 & 2,12 & 1144,74 & 3,2 \\
\hline 01-02 & 27,25 & 674,26 & 0,22 & 420,52 & 2,08 & 1124,32 & 3,2 \\
\hline $02-03$ & 25,81 & 638,81 & 0,21 & 398,41 & 1,97 & 1065,21 & 3,0 \\
\hline 03-04 & 25,58 & 633,09 & 0,21 & 394,84 & 1,95 & 1055,67 & 3,0 \\
\hline 04-05 & 24,24 & 599,81 & 0,20 & 374,08 & 1,85 & 1000,17 & 2,8 \\
\hline $05-06$ & 24,90 & 616,22 & 0,20 & 384,32 & 1,90 & 1027,55 & 2,9 \\
\hline $06-07$ & 24,68 & 610,70 & 0,20 & 380,88 & 1,88 & 1018,34 & 2,9 \\
\hline 07-08 & 29,02 & 718,10 & 0,23 & 447,86 & 2,21 & 1197,43 & 3,4 \\
\hline $08-09$ & 31,75 & 785,73 & 0,26 & 490,04 & 2,42 & 1310,19 & 3,7 \\
\hline $09-10$ & 39,76 & 983,98 & 0,32 & 613,68 & 3,03 & 1640,78 & 4,6 \\
\hline $10-11$ & 46,75 & 1157,03 & 0,38 & 721,61 & 3,57 & 1929,33 & 5,4 \\
\hline $\begin{array}{l}11-12 \\
12-13\end{array}$ & $\begin{array}{l}51,16 \\
52,09\end{array}$ & $\begin{array}{l}1265,99 \\
1288,98\end{array}$ & $\begin{array}{l}0,41 \\
0,42\end{array}$ & $\begin{array}{l}789,56 \\
803,91\end{array}$ & $\begin{array}{l}3,90 \\
3,97\end{array}$ & $\begin{array}{l}2111,03 \\
2149,37\end{array}$ & $\begin{array}{l}6,0 \\
6,1\end{array}$ \\
\hline $13-14$ & 58,03 & 1435,99 & 0,47 & 895,59 & 4,43 & 2394,50 & 6,8 \\
\hline $14-15$ & 50,70 & 1254,65 & 0,41 & 782,49 & 3,87 & 2092,11 & 5,9 \\
\hline $15-16$ & 45,92 & 1136,39 & 0,37 & 708,73 & 3,50 & 1894,92 & 5,4 \\
\hline $16-17$ & 43,90 & 1086,38 & 0,36 & 677,55 & 3,35 & 1811,54 & 5,1 \\
\hline $17-18$ & 37,67 & 932,26 & 0,30 & 581,42 & 2,87 & 1554,53 & 4,4 \\
\hline $18-19$ & 35,05 & 867,49 & 0,28 & 541,03 & 2,67 & 1446,54 & 4,1 \\
\hline $19-20$ & 34,12 & 844,39 & 0,28 & 526,62 & 2,60 & 1408,01 & 4,0 \\
\hline $20-21$ & 33,21 & 821,89 & 0,27 & 512,59 & 2,53 & 1370,50 & 3,9 \\
\hline $21-22$ & 31,47 & 778,69 & 0,25 & 485,65 & 2,40 & 1298,45 & 3,7 \\
\hline $22-23$ & 27,99 & 692,71 & 0,23 & 432,02 & 2,14 & 1155,09 & 3,3 \\
\hline $23-24$ & 29,28 & 724,59 & 0,24 & 451,91 & 2,23 & 1208,25 & 3,4 \\
\hline Total & 858,05 & 21234,63 & 6,95 & 13243,47 & 65,47 & 35408,57 & 100 \\
\hline
\end{tabular}

Las emisiones horarias generadas por las familias Anacardiaceae, Rubiaceae y Polygonaceae, corresponden al grupo de árboles que emiten desde 0,20 hasta $58,03 \mathrm{~kg} \mathrm{~h}^{-1}$, y presentan unos bajos niveles de emisión aunque tienen una gran abundancia y ocupan grandes áreas; esto se debe a que tienen factores de emisión relativamente bajos $\left(0,1\right.$ a $\left.0,6 \mu \mathrm{g} \mathrm{g}^{-1} \mathrm{~h}^{-1}\right)$.

La evolución horaria de las emisiones de monoterpenos de las familias Leguminosae y Euphorbiaceae en el PNNT, representa la mayor abundancia, aunado a que sus factores de emisión son responsables, en gran parte, de las emisiones de monoterpenos generadas en el $49,4 \%$ de área del PNNT. La familia Leguminosae es la mayor generadora, con aportes que llegan a 1435,99 $\mathrm{kg} \mathrm{h}^{-1}$ 
mientras que para Euphorbiaceae corresponden a 895,59 $\mathrm{kg} \mathrm{h}^{-1}$, ambas cuando se presentan los picos de temperatura de las 13-14 horas.

En general, la emisión de monoterpenos varía entre 1144,74 y $1310,19 \mathrm{~kg} \mathrm{~h}^{-1}$ durante las primeras horas del día, en la tarde luego de alcanzar el pico disminuyen de 2092,11 a $1554,53 \mathrm{~kg} \mathrm{~h}^{-1}$ (14-18 horas). Durante las primeras horas de la noche, sus tasas de emisión descienden de 1446,54 a 1298,45 $\mathrm{kg} \mathrm{h}^{-1}$ (18-22 horas) cuando la temperatura desciende hasta $26,8^{\circ} \mathrm{C}$.

Guenther et al. (1995), afirman que las emisiones biogénicas en el mundo se estiman en $1150 \mathrm{Tg} / a n ̃ o$ de las cuales el $11 \%$ de ellas corresponden a emisiones de monoterpenos (126,5 Tg/año), comparando este valor con el establecido en el presente estudio se puede inferir que corresponderían al $25 \%$ aproximadamente del total estimado para monoterpenos $(0,31 \mathrm{Tg} / \mathrm{año})$, siendo esta cantidad un valor considerable y que puede atribuirse principalmente a su posición geográfica, donde la incidencia de la radiación solar es constante a lo largo del año manteniendo temperaturas por encima del promedio anual de $28^{\circ} \mathrm{C}$.

El comportamiento de las tasas de emisión de monoterpenos está influenciado fuertemente por la temperatura, se observa que los picos se presentaron entre las 13 a 14 horas y los mínimos de 04 a 05 horas. En el periodo de tiempo comprendido entre las 00-07 horas se presenta una escasa variabilidad térmica con una desviación estándar de $\pm 0,5^{\circ} \mathrm{C}$.

\section{Comparación con otros inventarios}

La Tabla 4 relaciona inventarios realizados en zonas tropicales y subtropicales, estos inventarios realizados en zonas semitropicales bosques caducifolios de Monterrey (México) han establecido que las emisiones de monoterpenos son del orden de $1,33 \times 10^{-2} \mathrm{~kg} \mathrm{~km}^{-2} \mathrm{~d}^{-1}$ para condiciones similares al PNNT que presenta emisiones relativas de $1,1 \times 104 \mathrm{~kg} \mathrm{~km}^{-2} \mathrm{~d}^{-1}$.

Tabla 4. Comparación inventarios de emisiones en otras áreas tropicales

\begin{tabular}{lccc}
\hline \multicolumn{1}{c}{ Zona } & $\begin{array}{c}\text { Monoterpenos } \\
\left(\times 10^{3} \mathrm{~kg} \mathrm{~d}^{-1}\right)\end{array}$ & $\begin{array}{c}\text { Area promedio } \\
\left(\times 10^{3} \mathrm{~kg} \mathrm{~km}^{-2} \mathrm{~d}^{-1}\right)\end{array}$ & Referencia \\
\hline PNNT & $3,54 \times 10^{-1}$ & $1,13 \times 10^{-2}$ & Este estudio \\
Hong Kong & 9,56 & $8,69 \times 10^{-3}$ & $\begin{array}{c}\text { Chen et al. (2009) } \\
\text { Beijing }\end{array}$ \\
$\begin{array}{l}\text { Monterrey } \\
\text { Valle de Aburrá(Colombia) }\end{array}$ & 4,78 & $5,43 \times 10^{-4}$ & Whag, Yuhua \& Shuyu \\
& $4,3 \times 10^{1}$ & $1,33 \times 10^{-2}$ & $\begin{array}{c}\text { Gastelum (2000) } \\
\text { Toro, Cremades \& }\end{array}$ \\
\hline
\end{tabular}

Los inventarios relacionados en la tabla anterior, corresponden a zonas tropicales y subtropicales en épocas con temperatura promedio de $25-29^{\circ} \mathrm{C}$, donde se presentan emisiones similares a las generadas por las especies estudiadas en el PNNT con otras áreas donde hay bosques dominados por arboles caducifolios, así mismo, estas parecen ser más bajas en comparación con otras zonas donde existe presencia de vegetación de la familia Pinaceae, esto se debe a que estos árboles tiene factores de emisión mayores a $7 \mathrm{\mu g} \mathrm{g}^{-1} \mathrm{~h}^{-1}$ (Lancaster University, 2009). 


\section{CONCLUSIONES}

En total las emisiones biogénicas de monoterpenos en el PNNT son de $3,54 \times 10^{4} \mathrm{~kg}$ $\mathrm{d}^{-1}$. Estas emisiones están representadas en un $60 \%$ por la familia Leguminosae, siendo la mayor emisora, seguida de $37,4 \%$ por la familia Euphorbiaceae, 2,4\% para la familia Anacardiaceae y menos de $1 \%$ a las familias Rubiaceae y Polygonaceae.

Estudios realizados por el IDEAM, establecen que el PNNT se encuentra entre las zonas de Colombia con los valores más altos de radiación solar (entre 5,5 y 6,0 kWh $\mathrm{m}^{-2} \mathrm{~d}^{-1}$ ), esto indicaría un incremento en la temperatura y en consecuencia emisiones en las horas de mediodía (Spanke et al., 2001), coincidiendo con los resultados obtenidos en este estudio.

Este estudio y sus resultados presentan similitudes a los realizadas por Gustelum (2000), en la medida en que supone que las emisiones de monoterpenos son generados por vegetación caduca y su generación supone un proceso independiente de la luz.

Las emisiones de monoterpenos, al igual que el resto de compuestos biogénicos emitidos al aire, deben ser considerados como línea base al establecer si los precursores de contaminación fotoquímica de origen antropogénico deben de ser inspeccionados, minimizados o de ser posible evitar procesos productivos asociados con su generación, con la finalidad de reducir los niveles de ozono troposférico.

\section{AGRADECIMIENTOS}

Al Dr. José Antonio Adame y al Dr. Juan Pedro Bolívar Raya por sus orientaciones en el desarrollo de este proyecto de investigación. Al Posgrado Oficial en Tecnología Ambiental de la Universidad de Huelva por el apoyo en la realización de estos estudios. Al Departamento Administrativo de Ciencia, Tecnología e Innovación (COLCIENCIAS) en Colombia y a la Universidad del Magdalena por el financiamiento concedido en el marco del proyecto de investigación "Estimación de las Emisiones Biogénicas en el Departamento del Magdalena", a través del contrato RC No. 236-2008 y registrado con el código 1117-452-21217.

\section{REFERENCIAS}

- Arey, J., Atkinson, R. \& Aschmann, S. (1990). Product Study of the GasPhase Reactions of Monoterpenes with the $\mathrm{OH}$ Radical in the Presence of NOx. Journal of Geophysical Research, 95(D11), 18,539-18,546.

- Camargo, Y., Bolaño T., Álvarez A. (2010). Emisiones de compuestos orgánicos volátiles de origen Biogénico y su contribución a la dinámica atmosférica. Revista Intropica, 5, 77-86.

- Cremades, L. V. \& Ribes, S. (1997). Emisiones biogénicas procedentes de la vegetación terrestre en las áreas mediterráneas. II Jornadas sobre COVs. Emisión, Muestreo, Análisis y Tratamiento. Fórum Ambiental ECOMED POLLUTEC. Barcelona, 26 Febrero.

- Chen, F., Kin-Yin, J., Guenther, A. \& Wing-Kin, Y. (2009). A biogenic volatile organic compound emission inventory for Hong Kong. Atmospheric Environment, 43, 6442-6448. 
- Chuvieco, E. (2008). Teledetección Ambiental, la observación de la tierra desde el espacio. 3a ed. Madrid, España: Ariel.

- Derognat, C., Beekmann, M., Baeumle, M., Martin, D. \& Schmidt, H. (2003). Effect of biogenic volatile organic compound emissions on tropospheric chemistry during the Atmospheric Pollution Over the Paris Area (ESQUIF) campaign in the Ile-de-France region. Journal of Geophysical Research, 108 (D17), ESQ 3: 1-13.

- Fehsenfeld, F., Calvert, J., Fall, R., Goldan, P., Guenther, A. B., Hewitt, C. N., Lamb, B., Liu, S., Trainer, M., Westberg, H. \& Zimmerman, P. R. (1992). Emission of volatile organic compounds from vegetation and the implications for atmospheric chemistry. Global Biogeochemical Cycles, 6, 389-430.

- Gastelum, S. (2000). Estimación de las emisiones de isoprenos y monoterpenos generados por la vegetación que contribuyen a las condiciones de la cuenta atmosférica de Moterrey. Tesis Maestro en Ciencias Especialidad en Ciencias Ambientales. Programa de graduados en Ingeniería. Instituto Tecnológico y de Estudios Superiores de Monterrey, México.

- Guenther, A., Monson, R. K. \& Fall, R. (1991). Isoprene and monoterpene emission rate variability: observations with Eucalyptus and emission rate algorithm development. Journal of Geophysical Research, 96, 10799-10808.

- Guenther, A., Zimmerman, P. \& Harley, P. C. (1993). Isoprene and Monoterpene Emission Rate Variability: Model Evaluations and sensitivity Analyses. Journal of Geophysical Research, 98(D7), 12609-12617.

- Guenther, A., Zimmerman, P. \& Wildermuth. M. (1994). Natural volatile organic compound emission rate estimates for U.S. woodland landscapes. Atmospheric Environment, 28(6), 1197-1210.

- Guenther, A., Hewitt, C., Erickson, D., Fall, R., Geron, C., Graedel, T., Harley, P. et al. (1995). A global model of natural volatile organic compound emissions. Journal of Geophysical Research, 100(D5), 8873-8892.

- Instituto de Hidrología, Meteorología y Estudios Ambientales de Colombia IDEAM-. (2007a). Informe 151: Condiciones meteorológicas en agosto de 2007 y proyecciones en el corto, mediano y largo plazo. Bogotá D.C., Colombia.

- (2007b). Predicciones climáticas y alertas para planear y decidir. Bogotá D.C., Colombia. No. 151. Septiembre de 2007. ISSN 1909-3314.

- (2007c). Predicciones climáticas y alertas para planear y decidir. Bogotá D.C., Colombia. No. 152. Octubre de 2007. ISSN 1909-3314.

- Janson, R. W. (1992). Monoterpenes from the Boreal Coniferous Forest. Their Role in Atmospheric Chemistry. Doctoral Dissertation, Department of Meteorology, Stockholm University.

- Lancaster University. (2009). Database of Institute of Environmental and Natural Sciences, Department of Environmental Science. http://www.es.lancs.ac.uk/people/cnh, julio - 2009.

- Lerdau M., Litvak M. \& Monson, R. (1994). Plant-Chemical Defense Monoterpenes and the Growth-Differentiation Balance Hypothesis. Trends in Ecology \& Evolution, 9, 58-61.

- McGarvey, D. J. \& Croteau, R. (1995). Terpenoid metabolism. The Plant Cell, 7, 1015-1026.

- Monson, R. K., Lerdau, M. T., Sharkey, T. D., Schimel, D. S. \& Fall, R. (1995). Biological aspects of constructing volatile organic compound emissions inventories. Atmospheric Environment, 29, 2989-3002.

- Puentes, M. (2002). Plantas Parque Nacional Natural Tayrona. Las 100 especies más sobresalientes. Ministerio de Medio Ambiente. Delegación de la Comisión Europea para Colombia y Ecuador. Agencia Colombiana de Cooperación Internacional. Santa Marta. 
- Räisänen, T., Ryyppö, A. \& Kellomäki, S. (2009). Monoterpene emission of a boreal Scots pine (Pinus sylvestris L.) forest. Agric. For. Meteorol, 149, 808819.

- Sabillón, D. (2001). Determinación de los factores de emisión de Monoterpenos en tres especies típicas de la vegetación terrestre mediterránea: Pinus pinea, Pinus halepensis y Quercus ilex. Tesis doctoral. Departament d'Enginyeria Química. Universitat Politécnica de Catalunya. Barcelona, España.

- Schindler, T. \& Kotzias, D. (1989). Comparison of monoterpene volatilization and leaf-oil composition of conifers. Naturwissenschaften, 76, 475-476.

- Schuh, G., Heiden, A. C., Hoffmann, Th., Kahl, J., Rockel, P., Rudolph, J. \& Wildt, J. (1997). Emissions of Volatile Organic Compounds from Sunflower and Beech: Dependence on Temperature and Light Intensity. Journal of Atmospheric Chemistry, 27, 291-318.

- Simon, V., Clement, B., Riba, M.-L. \& Torres, L. (1994). The Landesexperiment: Monoterpenes emitted from the maritime pine. Journal of Geophysical Research, 99, 16501-16510.

- Spanke, J., Rannik, U., Forkel, R., Nigge, W. \& Hoffmann, T. (2001). Emission fluxes and atmospheric degradation of monoterpenes above a boreal forest: field measurements and modelling. Tellus Series B-Chemical and Physical Meteorology, 53, 406-422.

- Staudt, M. \& Bertin, N. (1998). Light and temperature dependence of the emission of cyclic and acyclic monoterpenes from holm oak (Quercus ilex L.) leaves. Plant, cell and Environment, 21, 385-395.

- Tao, Z., Larson, S. M., Wuebbles, D. J., Williams, A. \& Caughey, M. (2003). A seasonal simulation of biogenic contributions to ground-level ozone over the continental United States. Journal of Geophysical research, 108(D14), ACH 2: $1-15$.

- Thunis, P. \& Cavilier, C. (2000). Impact of biogenic emissions on ozone formation in the Mediterranean area- a BEMA modelling study. Atmospheric Environment, 34, 467-481.

- Tingey, D. T., Manning, M., Grothaus, L. C. \& Burns, W. F. (1980). Influence of light and temperature on monoterpene emissions from slash pine. Plant Physiology, 65, 797-801.

- Tingey, D. T., Turner, D. P. \& Weber, J. A. (1991). Factors Controlling the Emissions of Monoterpenes and Other Volatile Organic Compounds. En T. D. Sharkey, E. A. Holland \& H. A. Mooney (Eds.). Trace Gas Emissions By Plants (pp. 93-119). San Diego: Academic Press.

- Toro, G. M., Cremades O. L. \& Ramírez, B. J. (2001). Inventario de Emisiones Biogénicas en el Valle de Aburrá. Revista Ingeniería y Gestión Ambiental, 17(32-33).

- Velasco, E. (2003). Estimates for biogenic non-methane hydrocarbons and nitric oxide emissions in the Valley of Mexico. Atmospheric Environment, 37, 625-637.

- Velasco, E. \& Bernabé, R. (2004). Emisiones Biogénicas. México, D.F.: Col. Insurgentes Cuicuilco.

- Wang, Z., Yuhua, B. \& Shuyu, Z. (2003). A biogenic volatile organic compounds emission inventory for Beijing. Atmospheric Environment, 37, 3771-3782.

- Xu, Y., Wesely, M. L. \& Pierce, T. E. (2002). Estimates of biogenic emissions using satellite observations and influence of isoprene emission on $\mathrm{O} 3$ formation over the eastern United States. Atmospheric Environment, 36, 5819-5829. 
1. Magíster en Tecnología Ambiental. Grupo de Investigación en Modelación de Sistemas Ambientales -GIMSA-. Instituto de Investigaciones Tropicales INTROPIC-, Laboratorio No. 7, Universidad del Magdalena, Carrera 32 No. 22-08, 470003 - Santa Marta - Colombia. Tel. (+57) 54301292 ext. 273. gimsa@unimagdalena.edu.co

2. Magíster en Educación Ambiental. GIMSA. INTROPIC. ycamargo@unimagdalena.edu.co

3. Magíster en Tecnología Ambiental. GIMSA. INTROPIC. ing.andresvelez@gmail.com

Para citar este artículo: Bolaño Ortiz, T.R., Camargo Caicedo, Y. \& Vélez-Pereira, A.M. (2015). Emisiones biogénicas de monoterpenos en el Parque Nacional Natural Tayrona, Santa Marta (Colombia). Revista Luna Azul, 40, 102-116. Recuperado de http://lunazul.ucaldas.edu.co/index.php?option=content\&task=view\&id=1002 